\title{
Convergence Properties of the Symmetric and Unsymmetric Successive Overrelaxation Methods and Related Methods
}

\begin{abstract}
By David M. Young*
Abstract. The paper is concerned with variants of the successive overrelaxation method (SOR method) for solving the linear system $A u=b$. Necessary and sufficient conditions are given for the convergence of the symmetric and unsymmetric SOR methods when $A$ is symmetric. The modified SOR, symmetric SOR, and unsymmetric SOR methods are also considered for systems of the form $D_{1} u_{1}-C_{U} u_{2}=b_{1},-C_{L} u_{1}+D_{2} u_{2}=b_{2}$ where $D_{1}$ and $D_{2}$ are square diagonal matrices. Different values of the relaxation factor are used on each set of equations. It is shown that if the matrix corresponding to the Jacobi method of iteration has real eigenvalues and has spectral radius $\bar{\mu}<1$, then the spectral radius of the matrix $G$ associated with any of the methods is not less than that of the ordinary SOR method with $\omega=2\left(1+\left(1-\bar{\mu}^{2}\right)^{1 / 2}\right)^{-1}$. Moreover, if the eigenvalues of $G$ are real then no improvement is possible by the use of semi-iterative methods.
\end{abstract}

Introduction. In this paper we study convergence properties of several iterative methods for solving the linear system

$$
A u=b \text {, }
$$

where $A$ is a given real nonsingular $N \times N$ matrix with nonvanishing diagonal elements, $b$ is a given column vector, and $u$ is a column vector to be determined. Each method can be characterized by an equation

$$
u^{(n+1)}=g u^{(n)}+k, \quad n=0,1,2, \cdots,
$$

where $\mathcal{G}$ is the matrix associated with the particular iterative method and $k$ a constant column vector. It is easy to show that if all of the eigenvalues of $G$ are less than one in modulus and if $k=(I-G) A^{-1} b$, then for any $u^{(0)}$ the sequence $u^{(0)}, u^{(1)}, u^{(2)} \ldots$ converges to the solution of (1.1).

The successive overrelaxation method (SOR method), [15], is defined by the matrix

$$
\mathfrak{L}_{\omega}=(I-\omega L)^{-1}(\omega U+(1-\omega) I),
$$

where $L$ and $U$ are strictly lower and strictly upper triangular matrices, respectively, such that

$$
L+U=B=I-D^{-1} A
$$

Received October 31, 1969, revised March 2, 1970.

AMS 1969 subject classifications. Primary 6535; Secondary 1538, 6565.

Key words and phrases. Successive overrelaxation method, modified SOR, symmetric SOR, unsymmetric SOR, relaxation factor, spectral radius, virtual spectral radius, positive definite, $A^{1 / 2}$-norm, semi-iterative method, cyclic Chebyshev semi-iterative method.

* Work on this paper was supported in part by the National Science Foundation under grant GP-8442 and by the U. S. Army Research Office (Durham) under grant DA-ARO(D)-31-124-G1050 at the University of Texas at Austin. Partial support was also provided by the Office of Naval Research under Contract NO0014-67-A-0298-0015 at Harvard University. 
and $D=\operatorname{diag} A$ is the diagonal matrix with the same diagonal elements as $A$. Here $\omega$ is a real number known as a "relaxation factor" which is chosen in order that the convergence be as rapid as possible. Given $u^{(n)}$, the components of $u^{(n+1)}$ can be obtained by the SOR method one at a time in order from $u_{1}^{(n+1)}$ to $u_{N}^{(n+1)}$. This corresponds to a "forward sweep." One could also consider the use of a "backward sweep" where one determines in order the components from $u_{N}^{(n+1)}$ to $u_{1}^{(n+1)}$. D'Sylva and Miles [2] considered the unsymmetric SOR method (USSOR method) where each iteration consists of a forward sweep with relaxation factor $\omega$ followed by a backward sweep with relaxation factor $\tilde{\omega}$. The matrix associated with this iterative method is given by

$$
J_{\omega, \Phi}=\mathcal{U}_{\varpi} \mathfrak{L}_{\omega},
$$

where

$$
U_{\tilde{\omega}}=(I-\tilde{\omega} U)^{-1}(\tilde{\omega} L+(1-\tilde{\omega}) I) .
$$

As a special case, we have the symmetric SOR method (SSOR method) of Sheldon [11], where $\omega=\tilde{\omega}$. The SSOR method is defined by

$$
\mathfrak{S}_{\omega}=u_{\omega} \mathfrak{L}_{\omega} \text {. }
$$

Necessary and sufficient conditions for the convergence of the SOR, SSOR, and USSOR methods are given in Section 2. It is also shown that if $A$ is positive definite then $S\left(S_{\omega}\right)=\left\|\mathscr{L}_{\omega}\right\|_{A^{2} / . .}^{2}{ }^{* *}$

We consider generalization of the SOR, SSOR, and USSOR methods which are defined when $A$ has the form

$$
A=\left[\begin{array}{rr}
D_{1} & -C_{U} \\
-C_{L} & D_{2}
\end{array}\right),
$$

where $D_{1}$ and $D_{2}$ are square diagonal matrices. For the modified SOR method (MSOR method), [16], we use a relaxation factor $\omega$ for the equations corresponding to $D_{1}$ (called "red equations") and we use a possibly different relaxation factor $\omega^{\prime}$ for the equations corresponding to $D_{2}$ (called "black equations"). Thus, let us partition $u$ in accordance with (1.8) obtaining

$$
\left[\begin{array}{l}
u_{1}^{(n+1)} \\
u_{2}^{(n+1)}
\end{array}\right]=\mathcal{S}\left[\begin{array}{l}
u_{1}^{(n)} \\
u_{2}^{(n)}
\end{array}\right]+\left[\begin{array}{l}
k_{1} \\
k_{2}
\end{array}\right] .
$$

Letting $F=-D_{1}^{-1} C_{U}$ and $G=-D_{2}^{-1} C_{L}$, we have from (1.4)

$$
L+U=B=\left(\begin{array}{ll}
0 & F \\
G & 0
\end{array}\right) .
$$

For the MSOR method we obtain from (1.10) and (1.3) with the relaxation factors $\omega$ and $\omega^{\prime}$ the associated matrix

** In general, the spectral radius $S(G)$ of a matrix $G$ is the maximum of the moduli of its eigenvalues. Given a nonsingular matrix $P$ we define the $P$-norm of the matrix $G$ by $\|G\|_{P}=\left\|P G P^{-1}\right\|_{2}$. Here, for any real matrix $G$ the spectral norm of $G$ is defined by $\|G\|_{2}=\left(S\left(G G^{T}\right)\right)^{1 / 2}$. 


$$
\begin{aligned}
\mathscr{L}_{\omega, \omega^{\prime}} & =\left(\begin{array}{cc}
I_{1} & 0 \\
-\omega^{\prime} G & I_{2}
\end{array}\right)^{-1}\left[\begin{array}{cc}
(1-\omega) I_{1} & \omega F \\
0 & \left(1-\omega^{\prime}\right) I_{2}
\end{array}\right) \\
& =\left(\begin{array}{cc}
(1-\omega) I_{1} & \omega F \\
\omega^{\prime}(1-\omega) G & \omega \omega^{\prime} G F+\left(1-\omega^{\prime}\right) I_{2}
\end{array}\right) \\
& =\mathscr{L}_{0, \omega^{\prime}} \mathcal{L}_{\omega, 0},
\end{aligned}
$$

where $I_{1}$ and $I_{2}$ are identity submatrices.

The MSOR method with $\omega$ and $\omega^{\prime}$ fixed was first considered by DeVogelaere [1]. McDowell [9] considered more general variations of the relaxation factors and did not assume that $A$ had the form (1.8). Young, et al., [16], considered the MSOR method with $\omega$ and $\omega^{\prime}$ varying with $n$.

In Section 3 we give necessary and sufficient conditions for the convergence of the MSOR method, and we seek values of $\omega$ and $\omega^{\prime}$ to minimize $S\left(\mathcal{L}_{\left.\omega, \omega^{\prime}\right) \text { or }}\right.$ certain norms of $\mathscr{L}_{\omega, \omega}$, under certain restrictions on $\omega$ and $\omega^{\prime}$.

As a natural generalization of the USSOR method one can consider the use of the relaxation factors $\omega$ and $\omega^{\prime}$ on the forward sweep for the red and black equations, respectively, and then $\tilde{\omega}^{\prime}$ and $\tilde{\omega}$ on the backward sweep for the black and red equations, respectively. Thus, we have the unsymmetric modified SOR method (USMSOR method)

$$
W_{\omega, \omega^{\prime}, \tilde{\omega}, \tilde{\omega}^{\prime}}=U_{\tilde{\omega}, \tilde{\omega}^{\prime}} \mathfrak{L}_{\omega, \omega^{\prime}},
$$

where

$$
\begin{aligned}
\mathcal{U}_{\tilde{\omega}, \tilde{\omega}^{\prime}} & =\left[\begin{array}{cc}
I_{1} & -\tilde{\omega} F \\
0 & I_{2}
\end{array}\right)^{-1}\left[\begin{array}{cc}
(1-\tilde{\omega}) I_{1} & 0 \\
\tilde{\omega}^{\prime} G & \left(1-\tilde{\omega}^{\prime}\right) I_{2}
\end{array}\right) \\
& =\left(\begin{array}{cc}
(1-\tilde{\omega}) I_{1}+\tilde{\omega} \tilde{\omega}^{\prime} F G & \tilde{\omega}\left(1-\tilde{\omega}^{\prime}\right) F \\
\tilde{\omega}^{\prime} G & \left(1-\tilde{\omega}^{\prime}\right) I_{2}
\end{array}\right) \\
& =\mathfrak{L}_{\tilde{\omega}, 0} \mathfrak{L}_{0, \tilde{\omega}^{\prime}} \cdot
\end{aligned}
$$

As a special case we have the symmetric MSOR method*** (SMSOR method) defined by

$$
S_{\omega, \omega^{\prime}}=\mathcal{U}_{\omega, \omega^{\prime}} \mathfrak{L}_{\omega, \omega^{\prime}} .
$$

In Section 4 it is shown that if $A$ has the form (1.8), then the eigenvalues of each method considered can be expressed in terms of the eigenvalues of the modified SOR method for suitably defined relaxation factors. This generalizes the results of Wachspress [14] and D'Sylva and Miles [2] for the symmetric SOR method, and the results of Lynn [8] for the unsymmetric SOR method. It turns out that none of these methods can have a smaller spectral radius than the SOR method with the "optimum" relaxation factor [15]

$$
\omega_{b}=\frac{2}{1+\left(1-\bar{\mu}^{2}\right)^{1 / 2}}, \quad \bar{\mu}=S(B) .
$$

**** One could also consider the method defined by $\hat{S}_{\omega, \omega^{\prime}}=\mathcal{U}_{\omega^{\prime}, \omega^{\prime}} \mathcal{L}_{\omega, \omega^{\prime}}$. However, this method can be shown to have the same eigenvalues as $J_{\omega, \omega}$. 
In Section 5 we consider the possibility of accelerating the convergence of the methods by the use of semi-iteration [13]. It is shown that if $A$ is positive definite and has the form (1.8) and if all of the eigenvalues of $\mathfrak{L}_{\omega, \omega^{\prime}}$ are real, then the best semi-iteration method based on $\mathscr{L}_{\omega, \omega}$, is no better, as far as the spectral radius is concerned, than the best semi-iterative method based on the Gauss-Seidel method (whose matrix is $\mathscr{L}_{1,1} \equiv \mathscr{L}_{1}$ ). This latter semi-iterative method is referred to as the "GS-SI method." It follows that no semi-iterative method based on the USMSOR method can be more effective than the GS-SI method. We remark that the SOR method with the optimum relaxation factor $\omega_{b}$ has approximately the same rate of convergence as the GS-SI method.

In cases where $A$ does not have the form (1.8), on the other hand, one can often choose $\omega$ so that even though $S\left(\mathcal{S}_{\omega}\right)$ is considerably larger than $S\left(\mathcal{L}_{\omega_{\mathrm{b}}}\right)$, nevertheless, by the use of semi-iteration, one can obtain a significant improvement over the SOR method with $\omega=\omega_{b}$ (see Sheldon [11], Ehrlich [3], Evans and Forrington [4], and Habetler and Wachspress [6]).

For the case where $A$ does have the form (1.8), a comparison of the SOR method with $\omega=\omega_{b}$ and other methods is given in [17]. While the SOR method with $\omega=\omega_{b}$ is best as far as the spectral radius is concerned, as shown in [16], nevertheless, in terms of certain matrix norms the cyclic Chebyshev semi-iterative method introduced by Golub and Varga [5] and a modification of the GS-SI method proposed by Sheldon [11] are somewhat better.

2. The SOR, SSOR, and USSOR Methods. Let us first prove the following relation between the $A^{1 / 2}$-norms of the SSOR and SOR methods and the spectral radius of the SSOR method. We do not assume that $A$ necessarily has the form (1.8).

THEOREM 2.1. Let $A$ be a real symmetric matrix with positive diagonal elements. For any real $\omega$ the eigenvalues of $S_{\omega}$ are real and nonnegative. If $0<\omega<2$ and if $A$ is positive definite, then

$$
\left\|S_{\omega}\right\|_{A^{2} / 2}=S\left(\delta_{\omega}\right)=\left\|\mathscr{L}_{\omega}\right\|_{A^{2 / 2}}^{2}<1 .
$$

Conversely, if $S\left(S_{\omega}\right)<1$, then $0<\omega<2$ and $A$ is positive definite.

Proof. Since $\delta_{\omega}=\mathfrak{u}_{\omega} \mathfrak{L}_{\omega}$, it is easy to show, as in [17], that it is sufficient to consider the case where $A=I-L-U$ where $U=L^{T}$.

Evidently, by (1.7), (1.3), and (1.16), the matrix $\delta_{\omega}$ is similar to

$$
\delta_{\omega}^{*}=\left[(\omega L+(1-\omega) I)(I-\omega L)^{-1}\right]\left[(\omega L+(1-\omega) I)(1-\omega L)^{-1}\right]^{T},
$$

which is nonnegative definite. It follows that the eigenvalues of $\delta_{\omega}^{*}$ and hence, those of $S_{\omega}$ are real and nonnegative.

Suppose now that $A$ is positive definite and $0<\omega<2$ and let $\mathcal{G}^{\prime}=A^{1 / 2} \mathcal{G} A^{-1 / 2}$ for any matrix G. Following Wachspress [14] we have by (1.3)

$$
\mathfrak{L}_{\omega}^{\prime}=I-\omega A^{1 / 2}(I-\omega L)^{-1} A^{1 / 2}
$$

and

$$
\mathcal{L}_{\omega}^{\prime}\left(\mathcal{L}_{\omega}^{\prime}\right)^{T}=I-\omega(2-\omega)\left[A^{1 / 2}(I-\omega L)^{-1}\right]\left[A^{1 / 2}(I-\omega L)^{-1}\right]^{T} .
$$

Since $I-\mathscr{L}_{\omega}^{\prime}\left(\mathcal{L}_{\omega}^{\prime}\right)^{T}$ is positive definite for $0<\omega<2$, it follows that all eigenvalues of $\mathfrak{L}_{\omega}^{\prime}\left(\mathcal{L}_{\omega}^{\prime}\right)^{T}$ are less than unity and hence, 


$$
\left\|\mathscr{L}_{\omega}\right\|_{A^{2} / \%}=\left\|\mathscr{L}_{\omega}^{\prime}\right\|_{2}<1
$$

Evidently, $\delta_{\omega}$ is similar to

$$
\mathcal{S}_{\omega}^{\prime}=\mathcal{U}_{\omega}^{\prime} \mathcal{L}_{\omega}^{\prime} .
$$

But $\mathcal{u}_{\omega}^{\prime}=I-\omega A^{1 / 2}(I-\omega U)^{-1} A^{1 / 2}=\left(\mathscr{L}_{\omega}^{\prime}\right)^{T}$; hence,

$$
\left\|\mathscr{L}_{\omega}\right\|_{A^{2} / \mathrm{s}}^{2}=S\left(\mathcal{L}_{\omega}^{\prime}\left(\mathcal{L}_{\omega}^{\prime}\right)^{T}\right)=S\left(\mathcal{L}_{\omega}^{\prime} \mathcal{U}_{\omega}^{\prime}\right)=S\left(\mathcal{U}_{\omega}^{\prime} \mathcal{L}_{\omega}^{\prime}\right)=S\left(\mathcal{S}_{\omega}^{\prime}\right)=S\left(\mathcal{S}_{\omega}\right) .
$$

Moreover, $S_{\omega}^{\prime}=\left(\mathcal{L}_{\omega}^{\prime}\right)^{T} \mathcal{L}_{\omega}^{\prime}$ which is symmetric. Hence, $S\left(S_{\omega}^{\prime}\right)=\left\|\mathcal{S}_{\omega}^{\prime}\right\|_{2}=\left\|\mathcal{S}_{\omega}\right\|_{A^{1 / 2}}$ and (2.1) follows.

By (1.7), (1.3), and (1.6) we have

$$
\operatorname{det} S_{\omega}=(1-\omega)^{2 N} \text {. }
$$

Hence, if $S\left(\delta_{\omega}\right)<1$ then $0<\omega<2$. Moreover, $\delta_{\omega}=I-\omega(2-\omega)(I-\omega U)^{-1}$ $\cdot(I-\omega L)^{-1} A$ and $S_{\omega}$ is similar to $\delta_{\omega}^{* *}$, where

$$
\mathcal{S}_{\omega}^{* *}=I-\omega(2-\omega)(I-\omega L)^{-1} A(I-\omega U)^{-1} .
$$

If $A$ is not positive definite, then there exists a vector $v \neq 0$ and $\alpha \leqq 0$ such that $A v=\alpha v$. If $w=(I-\omega U) v$, then

$$
\frac{\left(w, S_{\omega}^{* *} w\right)}{(w, w)}=1-\omega(2-\omega) \alpha \frac{(v, v)}{(w, w)} \geqq 1,
$$

since $0<\omega<2$. But since $\delta_{\omega}^{* *}$ is symmetric, we have

$$
S\left(\mathcal{S}_{\omega}^{* *}\right)=\operatorname{Max}_{w \neq 0} \frac{\left(w, S_{\omega}^{* *} w\right)}{(w, w)} \geqq 1,
$$

and we have a contradiction. Therefore, $A$ must be positive definite, and the proof of Theorem 2.1 is complete.

For the USSOR method we have

THEOREM 2.2. Let $A$ be a real symmetric matrix with positive diagonal elements. If $0<\omega<2,0<\tilde{\omega}<2$, and if $A$ is positive definite, then

$$
S\left(J_{\omega, \tilde{\omega}}\right)<1 .
$$

On the other hand, if (2.3) holds, then

$$
0<\omega+\tilde{\omega}-\omega \tilde{\omega}<2 .
$$

Proof. By (1.5) we have

$$
\left\|J_{\omega, \tilde{\omega}}\right\|_{A^{2} / s}=\left\|u_{\tilde{\omega}} \mathcal{L}_{\omega}\right\|_{A^{1 / s}} \leqq\left\|\mathfrak{U}_{\tilde{\omega}}\right\|_{A^{1 / s}}\left\|\mathcal{L}_{\omega_{\omega}}\right\|_{A^{2 / s}} .
$$

If $0<\omega<2,0<\tilde{\omega}<2$, and if $A$ is positive definite, we have $\left\|\mathscr{L}_{\omega}\right\|_{A^{2} /,}<1$ by (2.1), and, similarly, $\left\|u_{\tilde{\omega}}\right\|_{A^{1 / 2}}<1$. Therefore, (2.3) holds.

Since det $J_{\omega, \Phi}=(1-\omega)^{N}(1-\tilde{\omega})^{N}$ and, since the product of the eigenvalues of $J_{\omega, \tilde{\omega}}$ is det $J_{\omega, \tilde{\omega}}$, it follows that $S\left(J_{\omega, \tilde{\omega}}\right) \geqq|(1-\omega)(1-\tilde{\omega})|$. If $(2.3)$ holds, then (2.4) holds.

It would be interesting to develop necessary conditions on the matrix $A$ in order that (2.3) holds either assuming that (2.4) holds or, perhaps, that $0<\omega<2$, $0<\tilde{\omega}<2$. It would also be interesting to show whether convergence would imply 
that $A$ is positive definite if (2.4) holds. As we shall see in Section 4, these propositions hold if $A$ has the form (1.8).

Using the $A^{1 / 2}$-norm we can give an alternative proof of a theorem of Ostrowski [10] concerning the convergence of the SOR method with variable $\omega$. A slightly weaker result was obtained by Wachspress [14] using the $A^{1 / 2}$-norm.

THEOREM 2.3. Let $A$ be a positive definite matrix. The SOR method using $\omega_{1}$, $\omega_{2}, \ldots$ converges provided either of the following conditions holds:

(a) for some $\epsilon>0$ we have

$$
\epsilon \leqq \omega_{k} \leqq 2-\epsilon
$$

for all $k$ sufficiently large:

(b) $0 \leqq \omega_{k} \leqq 2$ for all $k$ sufficiently large and the series

$$
\sum_{k=1}^{\infty} \omega_{k}\left(2-\omega_{k}\right)
$$

diverges.

Proof. For convergence it is clearly sufficient to show that

$$
\operatorname{Lim}_{m \rightarrow \infty}\left\|\prod_{k=m}^{1} \mathscr{L}_{\omega_{k}}\right\|_{A^{1 / \cdot}}=0 .
$$

Let $\nu(\omega)$ denote the smallest eigenvalue of the positive definite matrix

$$
P=\left(A^{1 / 2}(I-\omega L)^{-1}\right)\left(A^{1 / 2}(I-\omega L)^{-1}\right)^{T} .
$$

Evidently, $\nu(\omega)$ is a continuous function of $\omega$ since the eigenvalues of a matrix are continuous functions of its elements. Thus, there exists $\alpha>0$ such that $\nu(\omega) \geqq \alpha$ for all $\omega$ in the range $0 \leqq \omega \leqq 2$. From (2.2) we have

$$
\left\|\mathscr{L}_{\omega}\right\|_{A^{2} /,}^{2}=S\left(\mathfrak{L}_{\omega^{\prime}}^{\prime}\left(\mathcal{L}_{\omega}^{\prime}\right)^{T}\right) \leqq 1-\omega(2-\omega) \alpha .
$$

The proof is completed by noting that if conditions (a) or (b) hold, then (2.5) holds.

The proof given by Ostrowski [10] was based on the use of a certain quadratic form which is closely related to the $A^{1 / 2}$-norm. Thus, the proof given above and Ostrowski's proof are basically similar.

3. The MSOR Method. From [17] we have

THEOREM 3.1. Let $A$ be a matrix with nonvanishing diagonal elements of the form (1.8). Then:

(a) If $\mu$ is a nonzero eigenvalue of $B$ and if $\lambda$ satisfies

$$
(\lambda+\omega-1)\left(\lambda+\omega^{\prime}-1\right)=\omega \omega^{\prime} \lambda \mu^{2},
$$

then $\lambda$ is an eigenvalue of $\mathscr{L}_{\omega . \omega^{\prime}}$. If $\mu=0$ is an eigenvalue of $B$, then $\lambda=1-\omega$ and/or $\lambda=1-\omega^{\prime}$ is an eigenvalue of $\mathscr{L}_{\omega, \omega^{\prime}}$.

(b) If $\lambda$ is an eigenvalue of $\mathfrak{L}_{\omega, \omega^{\prime}}$, then there exists an eigenvalue $\mu$ of $B$ such that (3.1) holds.

For any $\mu$ let $\rho\left(\omega, \omega^{\prime}, \mu\right)$ be the root radius of the quadratic equation (3.1), i.e., the maximum of the moduli of the solutions $\lambda$ of (3.1). Evidently, we have

$$
S\left(\mathscr{L}_{\omega^{\prime}, \omega^{\prime}}\right)=\operatorname{Max}\left\{\operatorname{Max}_{\mu \neq 0 ; \mu \in S_{B}} \rho\left(\omega, \omega^{\prime}, \mu\right),|1-a|\right\},
$$


where

$a=1, \quad$ if $\quad \mu=0 \notin S_{B}$

$a=\omega, \quad$ if $\quad \mu=0 \in S_{B}$ and $1-\omega \in S_{\mathcal{L}_{\alpha} \ldots \ldots}$, but either

$$
1-\omega^{\prime} \notin S_{\mathcal{L}_{\omega} \ldots,} \text { or else }|1-\omega| \geqq\left|1-\omega^{\prime}\right| \text {, }
$$

$a=\omega^{\prime}, \quad$ if $\mu=0 \in S_{B}$ and $1-\omega^{\prime} \in S_{\mathcal{L}_{\omega, \ldots},}$, but either

$$
1-\omega \notin S_{\mathcal{L}_{\omega} \ldots}, \text { or else }\left|1-\omega^{\prime}\right| \geqq|1-\omega| \text {. }
$$

Here, for any matrix $G$ we let $S_{G}$ denote the set of all eigenvalues of $G$.

In most cases there are so many eigenvalues of $B$ that it is not practical to consider them individually. Rather, we consider bounds on $\mu$. We are thus led to define the virtual spectral radius of $\mathfrak{L}_{\omega, \omega}$ as

$$
\bar{S}\left(\mathscr{L}_{\omega, \omega^{\prime}}\right)=\underset{\mu}{\operatorname{LUB}} \rho\left(\omega, \omega^{\prime}, \mu\right),
$$

where $\mu$ ranges over the smallest convex set containing all of the eigenvalues of $B$. We say that $\mathscr{L}_{\omega, \omega^{\prime}}$ is strongly convergent if $\bar{S}\left(\mathscr{L}_{\omega, \omega^{\prime}}\right)<1$. In the case where the eigenvalues of $B$ are real, and where $A$ has the form (1.8), it can be shown [15] that $\mu$ is an eigenvalue of $B$ if and only if $-\mu$ is also an eigenvalue of $B$. Hence, we have

$$
\bar{S}\left(\mathfrak{L}_{\omega, \omega^{\prime}}\right)=\operatorname{Max}_{-\bar{\mu} \leq \mu \leq \bar{\mu}} \rho\left(\omega, \omega^{\prime}, \mu\right),
$$

where $\bar{\mu}=S(B)$.

THEOREM 3.2. Let $A$ be a real matrix with nonvanishing diagonal elements of the form (1.8). If $A$ is positive definite or, more generally, if the eigenvalues of $B$ are real and if $\bar{\mu}=S(B)<1$, then the MSOR method is strongly convergent if

$$
0<\omega<2, \quad 0<\omega^{\prime}<2 .
$$

Conversely, if the eigenvalues of $B$ are real and if the MSOR method is strongly convergent, then $\bar{\mu}<1$ and (3.3) holds. Also, if $A$ is symmetric and has positive diagonal elements and if the MSOR method is strongly convergent, then $A$ is positive definite and (3.3) holds.

Proof. We first state without proof the following lemma concerning the roots of a quadratic equation. (See, for instance, [18].)

LEMMA 3.3. If $b$ and $c$ are real, then the root radius of the quadratic equation

$$
\lambda^{2}-b \lambda+c=0
$$

is less than unity if and only if

$$
|c|<1, \quad|b|<1+c .
$$

It is shown in [15] that if $A$ is positive definite and has the form (1.8), then the eigenvalues of $B$ are real and $\bar{\mu}=S(B)<1$. Let us define

$$
c=(\omega-1)\left(\omega^{\prime}-1\right), \quad b=\omega \omega^{\prime} \mu^{2}-\omega-\omega^{\prime}+2=1+c-\omega \omega^{\prime}\left(1-\mu^{2}\right) .
$$

Evidently, if (3.3) holds, then (3.4) holds and the MSOR method is strongly convergent.

Suppose on the other hand that the MSOR method is strongly convergent and 
the eigenvalues of $B$ are real. Since $|c|<1$ and $|b|<1+c$ for all $\mu$ in the range $-\bar{\mu} \leqq \mu \leqq \bar{\mu}$, we have $\left|(\omega-1)\left(\omega^{\prime}-1\right)\right|<1$ and

$$
\left\{\begin{aligned}
\omega \omega^{\prime}\left(1-\mu^{2}\right) & >0, \\
(2-\omega)\left(2-\omega^{\prime}\right)+\omega \omega^{\prime} \mu^{2} & >0 .
\end{aligned}\right.
$$

Letting $\mu=0$, we get $\omega \omega^{\prime}>0$ and $(2-\omega)\left(2-\omega^{\prime}\right)>0$. Thus, (3.3) must hold. Since $\omega \omega^{\prime}>0$, it follows that all eigenvalues $\mu$ of $B$ are less than unity in modulus.

If $A$ is symmetric and has positive diagonal elements, then $B$ is similar to $I-$ $D^{-1 / 2} A D^{-1 / 2}$ and, hence, has real eigenvalues. If the MSOR method is strongly convergent, then $\bar{\mu}<1$ and, hence, $A$ is positive definite. Theorem 3.2 follows.

The following result was essentially proved in [16].

THEOREM 3.4. If $A$ is a real matrix with nonvanishing diagonal elements of the form (1.8) such that the eigenvalues of $B$ are real and $\bar{\mu}=S(B)<1$, then

$$
\bar{S}\left(\mathscr{L}_{\omega_{b}, \omega_{b}}\right)=S\left(\mathscr{L}_{\omega_{b}, \omega_{b}}\right)=S\left(\mathscr{L}_{\omega_{b}}\right)=\omega_{b}-1,
$$

and we have

$$
\bar{S}\left(\mathscr{L}_{\omega^{\prime} \omega^{\prime}}\right)>\bar{S}\left(\mathcal{L}_{\omega_{b}, \omega_{b}}\right),
$$

unless $\omega=\omega^{\prime}=\omega_{b}$.

We now seek an upper bound for $\bar{S}\left(\mathscr{L}_{\omega, \omega^{\prime}}\right)$ and also the values of $\omega$ and $\omega^{\prime}$ which minimize $\bar{S}\left(\mathscr{L}_{\omega, \omega^{\prime}}\right)$ subject to the conditions $0 \leqq \omega \leqq 1,0 \leqq \omega^{\prime} \leqq 1$. We prove

THEOREM 3.5. Under the hypotheses of Theorem 3.3 , if $0 \leqq \omega \leqq 1,0 \leqq \omega^{\prime} \leqq 1$, then

$$
S\left(\mathcal{L}_{\omega, \omega^{\prime}}\right)=\bar{S}\left(\mathcal{L}_{\omega, \omega^{\prime}}\right) \leqq 1-\frac{1}{2} \omega \omega^{\prime}\left(1-\bar{\mu}^{2}\right)
$$

and

$$
\bar{S}\left(\mathscr{L}_{\omega, \omega^{\prime}}\right)=S\left(\mathscr{L}_{\omega, \omega^{\prime}}\right)>S\left(\mathscr{L}_{1,1}\right)=\bar{S}\left(\mathscr{L}_{1,1}\right)=\bar{\mu}^{2},
$$

unless $\omega=\omega^{\prime}=1$.

Proof. We first state without proof the following lemma.

Lemma 3.6. Let $\rho$ and $\rho^{\prime}$ be the root radii of $\lambda^{2}-b \lambda+c=0$ and $\lambda^{2}-b^{\prime} \lambda+$ $c=0$, where $b, c$, and $b^{\prime}$ are real. If $|b| \geqq\left|b^{\prime}\right|$, then $\rho \geqq \rho^{\prime}$. Moreover, if $\left(b^{\prime}\right)^{2}-$ $4 c \geqq 0$ and $|b|>\left|b^{\prime}\right|$, then $\rho>\rho^{\prime}$.

Since $b=1+c-\omega \omega^{\prime}\left(1-\mu^{2}\right)$ from (3.5), and since $0 \leqq \omega \leqq 1,0 \leqq \omega^{\prime} \leqq 1$, $c=(\omega-1)\left(\omega^{\prime}-1\right)$, the largest value of $|b|$ in the range $-\bar{\mu} \leqq \mu \leqq \bar{\mu}$, occurs, for fixed $\omega$ and $\omega^{\prime}$, where $\mu=\bar{\mu}$. Consequently, by Lemma 3.6 we have, since $\bar{\mu}$ is an eigenvalue of $B$,

$$
\bar{S}\left(\mathscr{L}_{\omega, \omega^{\prime}}\right)=\rho\left(\omega, \omega^{\prime}, \bar{\mu}\right)=S\left(\mathscr{L}_{\omega^{\prime} \omega^{\prime}}\right) .
$$

We now seek a bound on $\rho\left(\omega, \omega^{\prime}, \bar{\mu}\right)$. Letting $\theta=1-\lambda$, we have from (3.5), with $\mu=\bar{\mu}$, that $\lambda^{2}-b \lambda+c=0$ becomes

$$
\theta^{2}-\left(1+\omega \omega^{\prime}\left(1-\bar{\mu}^{2}\right)-c\right) \theta+\omega \omega^{\prime}\left(1-\bar{\mu}^{2}\right)=0 .
$$

But the discriminant of $(3.10)$ is

$$
\left(\omega-\omega^{\prime}\right)^{2}+2 \bar{\mu}^{2}\left(2-\left(\omega+\omega^{\prime}\right) \omega \omega^{\prime}\right),
$$

which is nonnegative since $0 \leqq \omega \leqq 1,0 \leqq \omega^{\prime} \leqq 1$. Since $0 \leqq c<1$, the smallest 
root of (3.10) is not less than

$$
\frac{1}{2} \omega \omega^{\prime}\left(1-\bar{\mu}^{2}\right) .
$$

Hence, (3.8) follows.

Let $c$ be any number in the range $0<c<1$. It is easy to show that $b=1+$ $c-\omega \omega^{\prime}\left(1-\bar{\mu}^{2}\right)$ is minimized, subject to the conditions $0 \leqq \omega \leqq 1,0 \leqq \omega^{\prime} \leqq 1$, $(\omega-1)\left(\omega^{\prime}-1\right)=c$ when

$$
\omega=\omega^{\prime}=\omega_{0}=1-\sqrt{c} .
$$

Therefore, by Lemma 3.6 we have

$$
\rho\left(\omega, \omega^{\prime}, \bar{\mu}\right) \geqq \rho(1-\sqrt{c}, 1-\sqrt{c}, \bar{\mu}) .
$$

Moreover, $\rho\left(1-\sqrt{c}, 1-\sqrt{c}, \bar{\mu}^{2}\right)$ is the root radius of

$$
\lambda^{2}-\left(1+c-(1-\sqrt{c})^{2}\left(1-\bar{\mu}^{2}\right)\right) \lambda+c=0,
$$

i.e.,

$$
\left(\lambda+\omega_{1}-1\right)^{2}=\omega_{1}^{2} \bar{\mu}^{2} \lambda,
$$

where $\omega_{1}=1-\sqrt{c}$. The root radius is

$$
\rho\left(\omega_{1}\right)=\left[\frac{\omega_{1} \bar{\mu}+\left(\omega_{1}^{2} \bar{\mu}^{2}-4\left(\omega_{1}-1\right)\right)^{1 / 2}}{2}\right]^{2}
$$

which can be shown to be a decreasing function of $\omega_{1}$ for $0 \leqq \omega_{1} \leqq 1$. Therefore, unless $\omega=\omega^{\prime}=1$ we have $S\left(\mathscr{L}_{\omega, \omega^{\prime}}\right)>S\left(\mathscr{L}_{1,1}\right)$. Since $\rho\left(1,1, \vec{\mu}^{2}\right)=\bar{\mu}^{2}$, the result (3.9) follows.

Let us assume that $A$ is positive definite and has the form (1.8). We shall study the behavior of the $D^{1 / 2}$-norm and the $A^{1 / 2}$-norm of $\mathcal{L}_{\omega, \omega}$. Actually, we shall consider the virtual $D^{1 / 2}$-norm and the virtual $A^{1 / 2}$-norm defined by

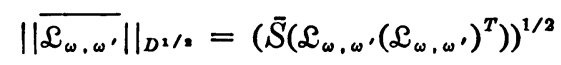

and

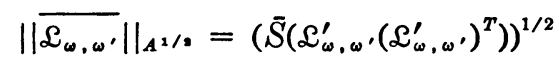

respectively, where $\mathscr{L}_{\omega^{\prime} \omega^{\prime}}^{\prime}=A^{1 / 2} \mathscr{L}_{\omega, \omega^{\prime}} A^{-1 / 2}$.

Concerning the virtual $D^{1 / 2}$-norm we state the following result.

If $\bar{\mu}>(1 / 3)^{1 / 2} \doteq .577$, then $\left\|\overline{\mathscr{L}_{\omega, \omega}}\right\|_{D^{1 / 2}}$ is minimized for

$$
\omega=\frac{4}{5+\bar{\mu}^{2}}, \quad \omega^{\prime}=\frac{4}{3-\bar{\mu}^{2}}
$$

and

$$
\underset{\omega, \omega^{\prime}}{\operatorname{Min}}\left\|\overline{\mathscr{L}_{\omega, \omega^{\prime}}}\right\|_{D^{1 / \rho}}=\frac{1+\bar{\mu}^{2}}{3-\bar{\mu}^{2}} .
$$

A proof of this result will be given in a later paper.

Concerning the $A^{1 / 2}$-norm, we have the following

THEOREM 3.7. If $A$ is a positive definite matrix of the form (1.8), then

$$
\left\|\overline{\mathscr{Q}_{1,1}}\right\|_{A^{2} / \ell}=\left\|\mathscr{L}_{1,1}\right\|_{A^{2} /,}=\bar{\mu}
$$


and we have

$$
\left\|\overline{\mathfrak{L}_{\omega, \omega} \cdot}\right\|_{A^{1 / 2}}>\left\|\mathfrak{L}_{1,1}\right\|_{A^{1 / 2}},
$$

unless $\omega=\omega^{\prime}=1$ or $\bar{\mu}=0$. Moreover, for any $\omega$ and $\omega^{\prime}$ in the range $0 \leqq \omega \leqq 2$, $0 \leqq \omega^{\prime} \leqq 2$ we have

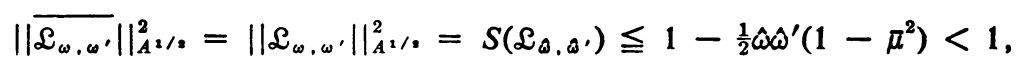

where

$$
\hat{\omega}=\omega(2-\omega), \quad \hat{\omega}^{\prime}=\omega^{\prime}\left(2-\omega^{\prime}\right) .
$$

Proof. As shown in [15], we can assume that $\operatorname{diag} A=I$. From (1.11) and (1.13) we have

$$
\begin{aligned}
\mathscr{L}_{\omega^{\prime} \omega^{\prime}}^{\prime} & =A^{1 / 2} \mathscr{L}_{\omega, \omega^{\prime}} A^{-1 / 2} \\
& =A^{1 / 2}\left[I-\left[\begin{array}{cc}
\omega I_{1} & 0 \\
\omega \omega^{\prime} G & \omega^{\prime} I_{2}
\end{array}\right] A\right] A^{-1 / 2}=I-A^{1 / 2}\left(\begin{array}{cc}
\omega I_{1} & 0 \\
\omega \omega^{\prime} G & \omega^{\prime} I_{2}
\end{array}\right) A^{1 / 2}
\end{aligned}
$$

and

$$
\mathcal{U}_{\omega, \omega^{\prime}}^{\prime}=I-A^{1 / 2}\left(\begin{array}{cc}
\omega I_{1} & \omega \omega^{\prime} F \\
0 & \omega^{\prime} I_{2}
\end{array}\right) A^{1 / 2} .
$$

Since $F=G^{T}$ we have $\left(\mathcal{L}_{\omega, \omega}^{\prime}\right)^{T}=\mathfrak{U}_{\omega, \omega^{\circ}}^{\prime}$ and

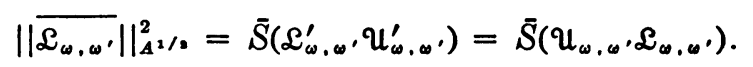

We now prove

LEMMA 3.8. If $A$ is a matrix of the form (1.8) whose diagonal elements do not

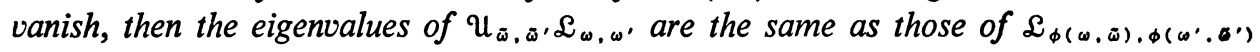
where, in general, $\phi\left(\omega_{1}, \omega_{2}\right)=\omega_{1}+\omega_{2}-\omega_{1} \omega_{2}$.

Proof. This is a generalization of a result proved by Wachspress [14, pp. 162-163]. By (1.11) and (1.13) we have

$$
\mathfrak{U}_{\tilde{\omega}, \varpi}, \mathfrak{L}_{\omega, \omega}=\mathfrak{L}_{\varpi, 0} \mathfrak{L}_{0, \bar{\omega}}, \mathfrak{L}_{0, \omega}, \mathfrak{L}_{\omega, 0}
$$

which has the same eigenvalues as

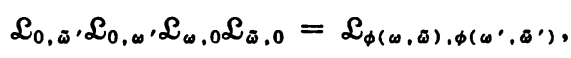

and the lemma follows.

Applying Lemma 3.8 and using (3.15), we obtain

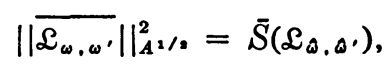

where $\hat{\omega}$ and $\hat{\omega}^{\prime}$ are given by (3.14). Therefore,

$$
\left\|\overline{\mathfrak{L}_{1,1}}\right\|_{A^{1 / 2}}^{2}=\bar{S}\left(\mathfrak{L}_{1,1}\right)=\bar{S}\left(\mathfrak{L}_{1}\right)=S\left(\mathfrak{L}_{1}\right)=\bar{\mu}^{2},
$$

hence, (3.11) follows. Moreover, since $0<\omega<2$ and $0<\omega^{\prime}<2$, if the MSOR method is strongly convergent, it follows that $0<\hat{\omega} \leqq 1,0 \leqq \hat{\omega}^{\prime} \leqq 1$. By (3.8), the result (3.13) follows. The result (3.12) follows from Theorem 3.5, and the proof of Theorem 3.7 is complete.

From Lemma 3.8 it follows that the eigenvalues of $\delta_{\omega}$ are the same as those 


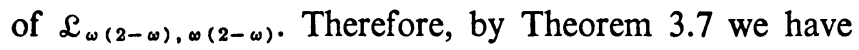

$$
S\left(\mathcal{S}_{\omega}\right)=\left\|\mathscr{L}_{\omega}\right\|_{A^{2} / \mathrm{s}}^{2}=\left\|\overline{\mathcal{L}_{\omega, \omega}}\right\|_{A^{2} / \mathrm{s}}^{2} \geqq\left\|\mathscr{L}_{1,1}\right\|_{A^{2} / \mathrm{s}}^{2}=\bar{\mu}^{2}=S\left(\mathscr{L}_{1,1}\right)=S\left(\mathcal{S}_{1}\right) .
$$

The fact that $S\left(S_{\omega}\right) \geqq S\left(S_{1}\right)$ for positive definite matrices of the form (1.8) was proved by Kahan [7, Chapter 5].

Using (3.13) we can prove the following generalization of Theorem 2.2.

THEOREM 3.9. Let $A$ be a real positive definite matrix of the form (1.8). The MSOR method with $\omega_{1}, \omega_{1}^{\prime}, \omega_{2}, \omega_{2}^{\prime}, \cdots$ converges provided either of the following conditions holds:

(a) for some $\epsilon>0$ we have

$$
\epsilon \leqq \omega_{k} \leqq 2-\epsilon, \quad \epsilon \leqq \omega_{k}^{\prime} \leqq 2-\epsilon,
$$

for all $k$ sufficiently large,

(b) $0 \leqq \omega_{k} \leqq 2$ and $0 \leqq \omega_{k}^{\prime} \leqq 2$ for all $k$ sufficiently large and the series

$$
\sum_{k=1}^{\infty} \omega_{k} \omega_{k}^{\prime}\left(2-\omega_{k}\right)\left(2-\omega_{k}^{\prime}\right)
$$

diverges.

4. The USMSOR Method. In this section we consider the USMSOR method and special cases thereof including the SMSOR, USSOR, and SSOR methods. From (1.12) and Theorem 3.7 it follows that

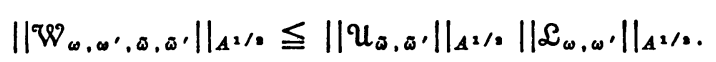

Hence, we have

THEOREM 4.1. If $A$ is a positive definite matrix of the form (1.8) and if $0<\omega<2$, $0<\omega^{\prime}<2,0<\tilde{\omega}<2,0<\tilde{\omega}^{\prime}<2$, then

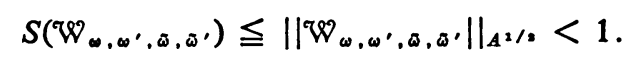

From (1.12) and Lemma 3.8 we have

THEOREM 4.2. Let $A$ be a real symmetric matrix of the form (1.8) with positive diagonal elements. The eigenvalues of ${ }^{W_{\omega}, \omega^{\prime}, \tilde{\omega}, \tilde{\omega}^{\prime}}$ are the same as those of $\mathcal{L}_{\omega, \omega^{\prime}}$ where

$$
\hat{\omega}=\omega+\bar{\omega}-\omega \tilde{\omega}, \quad \hat{\omega}^{\prime}=\omega^{\prime}+\bar{\omega}^{\prime}-\omega^{\prime} \bar{\omega}^{\prime} .
$$

We now define the virtual spectral radius of $W_{\omega, \omega^{\prime}, \tilde{\omega}, \tilde{\omega}^{\prime}}$ by

$$
\bar{S}\left(W_{\omega, \omega^{\prime}, \tilde{\omega}, \bar{\omega}^{\prime}}\right)=\bar{S}\left(\mathscr{L}_{\hat{\omega}^{\prime} \hat{\omega}^{\prime}}\right) \text {, }
$$

where $\hat{\omega}$ and $\hat{\omega}^{\prime}$ are given by (4.2).

THEOREM 4.3. Under the hypotheses of Theorem 4.2, if $A$ is positive definite and if $0<\hat{\omega}<2,0<\hat{\omega}^{\prime}<2$, where $\hat{\omega}$ and $\hat{\omega}^{\prime}$ are given by (4.2), then

$$
S\left(W_{\omega, \omega^{\prime}, \tilde{\omega}, \omega^{\prime}}\right) \leqq \bar{S}\left(W_{\omega, \omega^{\prime}, \tilde{\omega}, \tilde{\omega}^{\prime}}\right)<1 .
$$

Moreover, if $\omega \neq 1, \omega^{\prime} \neq 1$, then

$$
\bar{S}\left(W_{\omega_{0}, \omega^{\prime}, \psi(\omega), \psi\left(\omega^{\prime}\right)}\right)=S\left(\mathscr{L}_{\omega_{b}, \omega_{b}}\right)=S\left(\mathscr{L}_{\omega_{b}}\right)=\omega_{b}-1,
$$

where $\psi(\omega)$ is defined for $\omega \neq 1$ by 


$$
\psi(\omega)=\frac{\omega_{b}-\omega}{1-\omega},
$$

where $\omega_{b}$ is given by (1.15) and $\psi(1)=1$ if $\omega_{b}=1$. If $\omega_{b} \neq 1$, then

$$
\bar{S}\left(\mathscr{W}_{\omega, \omega^{\prime}, \tilde{\omega}, \tilde{\omega}^{\prime}}\right)>\omega_{b}-1 \text {, }
$$

unless $\omega \neq 1, \omega^{\prime} \neq 1, \tilde{\omega}^{\prime}=\psi\left(\omega^{\prime}\right), \tilde{\omega}=\psi(\omega)$. If $\omega_{b}=1$, then (4.7) holds unless $\omega$ or $\tilde{\omega}=1$ and unless $\omega^{\prime}=1$ or $\tilde{\omega}^{\prime}=1$. Conversely, if (4.4) holds, then $A$ is positive definite and $0<\hat{\omega}<2,0<\hat{\omega}^{\prime}<2$.

Proof. By (4.3) and Theorem 3.4 we minimize $\bar{S}\left(W_{\omega, \omega^{\prime}, \omega_{.} \omega^{\prime}}\right)$ by letting $\hat{\omega}=$ $\hat{\omega}^{\prime}=\omega_{b}$, i.e., by letting

$$
\omega+\tilde{\omega}-\omega \tilde{\omega}=\omega^{\prime}+\tilde{\omega}^{\prime}-\omega^{\prime} \tilde{\omega}^{\prime}=\omega_{b} .
$$

If (4.2) holds, then by (4.3) it follows that $\bar{S}\left(\mathscr{S}_{\hat{\Theta}_{,}}\right)<1$. Since $A$ is real and symmetric and has positive diagonal elements, it follows from Theorem 3.2 that $A$ is positive definite and $0<\hat{\omega}<2,0<\hat{\omega}^{\prime}<2$.

From Theorem 4.3 it follows that no choice of $\omega, \omega^{\prime}, \tilde{\omega}, \tilde{\omega}^{\prime}$ can yield a faster convergence, as far as the spectral radius is concerned, than the SOR method with $\omega=\omega_{b}$. This result was proved for the USSOR method by D'Sylva and Miles [2].

From Theorem 4.2 it follows that for the SMSOR method, the eigenvalues of $\delta_{\omega, \omega}$ are the same as those of

$$
\mathcal{L}_{\omega(2-\omega), \omega^{\prime}\left(2-\omega^{\prime}\right)} .
$$

Consequently, by Theorem 3.5 the optimum choice of $\omega$ and $\omega^{\prime}$ is $\omega=\omega^{\prime}=1$.

For the USSOR method, the eigenvalues of $J_{\omega, \tilde{\omega}}$ are the same as those of $\mathfrak{L}_{\omega+\Phi-\omega \otimes}$. One can obtain a spectral radius of $\omega_{b}-1$ by letting $\omega \neq 1$, if $\omega_{b} \neq 1$, and letting $\bar{\omega}=\left(\omega_{b}-\omega\right)(1-\omega)^{-1}$. If $\omega_{b}=1$ we can let $\omega=1$ or $\tilde{\omega}=1$.

5. Semi-iterative Methods. In this section we consider semi-iterative methods based on the MSOR method and the methods considered in Section 4. We assume that the matrix $A$ has the form (1.8). Since the eigenvalues of the methods of Section 4 are related to those of $\mathscr{L}_{\omega, \omega}$, for suitable $\omega$ and $\omega^{\prime}$, it is sufficient to study the eigenvalues of $\mathfrak{L}_{\omega, \omega^{\prime}}$.

Given a basic iterative method

$$
u^{(n+1)}=g u^{(n)}+k,
$$

where the eigenvalues of $\mathcal{G}$ are real and lie in an interval $\alpha \leqq \lambda \leqq \beta<1$, we can accelerate the convergence by using a semi-iterative method. The convergence of the semi-iterative method depends on the quantity

$$
\sigma=\frac{\beta-\alpha}{2-(\beta+\alpha)},
$$

where the smaller $\sigma$ the faster the convergence (see, for instance, Varga [13] or [17]). For the Gauss-Seidel method we have $\beta=\bar{\mu}^{2}, \alpha=0$ and hence,

$$
\sigma=\frac{\bar{\mu}^{2}}{2-\bar{\mu}^{2}} .
$$


THEOREM 5.1. Let $A$ be a positive definite matrix of the form (1.8) and let $\bar{\mu}=S(B)$. If the solutions of (3.1) are real for all $\mu$ in the interval $-\bar{\mu} \leqq \mu \leqq \bar{\mu}$, then

$$
\sigma=\sigma\left(\omega, \omega^{\prime}\right)=\frac{\beta-\alpha}{2-(\beta+\alpha)} \geqq \frac{\bar{\mu}^{2}}{2-\bar{\mu}^{2}}=\sigma(1,1) .
$$

Hence, $\beta$ and $\alpha$ are, respectively, the maximum and minimum of all roots of (3.1) when $\mu$ assumes all values between $-\bar{\mu}$ and $\bar{\mu}$. Moreover, if $\omega^{\prime}=1$ and $1 \leqq$ $\omega \leqq\left(1-\bar{\mu}^{2}\right)^{-1}$ or if $\omega=1$ and $1 \leqq \omega^{\prime} \leqq\left(1-\bar{\mu}^{2}\right)^{-1}$, then

$$
\sigma\left(\omega, \omega^{\prime}\right)=\sigma(1,1)=\frac{\bar{\mu}^{2}}{2-\bar{\mu}^{2}} .
$$

We now sketch a proof of the theorem. Details of the proof can be found in [18].

Figure 5.1 shows the regions of the $\left(\omega, \omega^{\prime}\right)$ plane where all roots of $(3.1)$ are real for all $\mu$ in the interval $-\bar{\mu} \leqq \mu \leqq \bar{\mu}$. Thus, all roots are real unless we have

$$
\omega>\frac{1}{1-\mu^{2}}, \quad \omega^{\prime}>\omega\left(\frac{1}{(\omega-1)^{1 / 2} \bar{\mu}+\left(1-\bar{\mu}^{2}\right)^{1 / 2}}\right)^{2},
$$

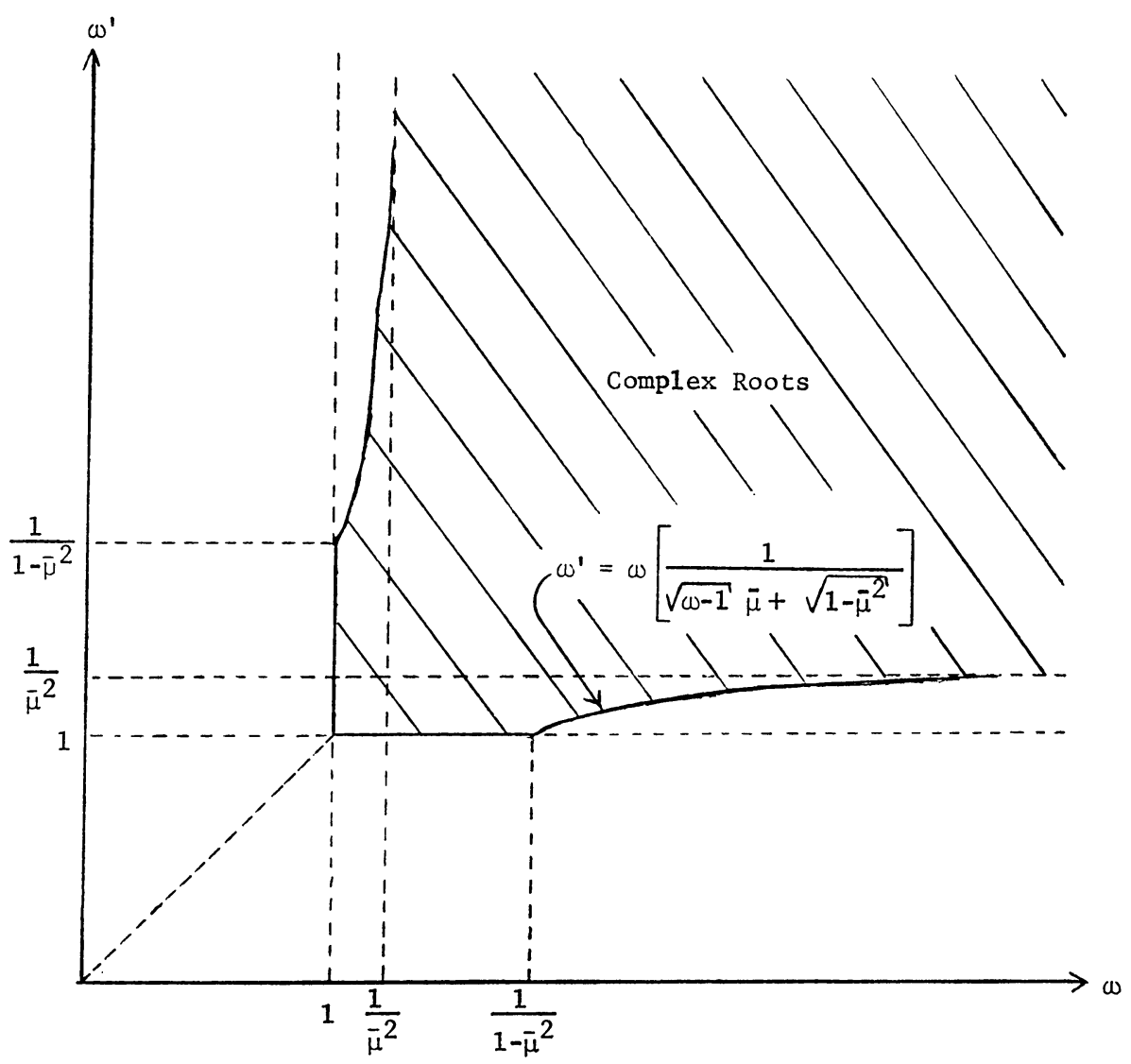

Figure 5.1. Regions of Real and Complex Eigenvalues of $\mathfrak{L}_{\omega, \omega}$. 


$$
\begin{gathered}
\omega^{\prime}>\frac{1}{1-\bar{\mu}^{2}}, \quad \omega>\omega^{\prime}\left(\frac{1}{(\omega-1)^{1 / 2} \bar{\mu}+\left(1-\bar{\mu}^{2}\right)^{1 / 2}}\right)^{2} . \\
1<\omega \leqq \frac{1}{1-\bar{\mu}^{2}}, \quad 1<\omega^{\prime} \leqq \frac{1}{1-\bar{\mu}^{2}} .
\end{gathered}
$$

Theorem 5.1 is proved by considering the following cases.

Case (a): $\omega^{\prime}=1$.

$$
\begin{aligned}
\sigma\left(\omega, \omega^{\prime}\right) & =\frac{1-\omega\left(1-\bar{\mu}^{2}\right)}{1+\omega\left(1-\bar{\mu}^{2}\right)}>\frac{\bar{\mu}^{2}}{2-\bar{\mu}^{2}}, & & \text { if } \quad \omega<1, \\
& =\frac{\bar{\mu}^{2}}{2-\bar{\mu}^{2}}, & & \text { if } \quad 1 \leqq \omega \leqq \frac{1}{1-\bar{\mu}^{2}}, \\
& =\frac{\omega-1}{\omega+1}>\frac{\bar{\mu}^{2}}{2-\bar{\mu}^{2}}, & & \text { if } \quad \omega>\frac{1}{1-\bar{\mu}^{2}} .
\end{aligned}
$$

Case (b): $\quad \omega=\omega^{\prime}<1$.

$$
\sigma(\omega, \omega)=\left(1-\frac{4\left(1-\bar{\mu}^{2}\right)}{\left(2-\omega \bar{\mu}^{2}\right)^{2}}\right)^{1 / 2}>\frac{\bar{\mu}^{2}}{2-\bar{\mu}^{2}} .
$$

Case (c): $\omega^{\prime} \leqq \omega<1$.

$$
\sigma\left(\omega, \omega^{\prime}\right) \geqq \sigma(\hat{\omega}, \hat{\omega})>\frac{\bar{\mu}^{2}}{2-\bar{\mu}^{2}}, \quad \text { where } \quad \hat{\omega}=1-\left((1-\omega)\left(1-\omega^{\prime}\right)\right)^{1 / 2} .
$$

Case (d): $\omega \geqq \omega^{\prime}>1$.

$$
\sigma\left(\omega, \omega^{\prime}\right)=\frac{\omega-\omega^{\prime}}{\omega+\omega^{\prime}} \geqq \frac{\omega-\omega\left[(\omega-1)^{1 / 2} \bar{\mu}+\left(1-\bar{\mu}^{2}\right)^{1 / 2}\right]^{-2}}{\omega+\omega\left[(\omega-1)^{1 / 2} \bar{\mu}+\left(1-\bar{\mu}^{2}\right)^{1 / 2}\right]^{-2}} \geqq \frac{\bar{\mu}^{2}}{2-\bar{\mu}^{8}} .
$$

Case (e): $\omega^{\prime}<1, \omega>1$.

$$
\sigma\left(\omega, \omega^{\prime}\right) \geqq \sigma\left(\frac{\omega}{\omega^{\prime}}, 1\right) \geqq \frac{\bar{\mu}^{2}}{2-\bar{\mu}^{2}} .
$$

It follows from Theorems 4.2 and 5.1 that if $A$ is positive definite and has the form (1.8) then we cannot achieve any faster convergence than that of the GS-SI method by using any semi-iterative method based on $W_{\omega, \infty}{ }_{\omega, \sigma} \cdots$ Mereover, the GS-SI method does not converge faster than the SOR method with $\omega=\omega_{b}$. The above statements are based on the use of the spectral radius as a measure of convergence. As discussed in Section 1, different conclusions may be appropriate if one uses certain matrix norms as a measure of convergence.

Acknowledgments. The author is pleased to acknowledge the assistance of David R. Kincaid of The University of Texas at Austin. The helpful suggestions of the referees, especially on Theorems 2.3 and 3.9 are also acknowledged. 
1. R. DeVogelaere, "Overrelaxations," Notices Amer. Math. Soc., v. 5, 1958, p. 147. Abstract \#539-53.

2. E. D'SyLva \& G. A. Miles, "The S.S.O.R. iteration scheme for equations with $\sigma_{1}$-ordering," Comput. J., v. 6, 1963/64, pp. 366-367. MR 28 \#1772.

3. Louis W. EHRLICH, "The block symmetric successive overrelaxation method," J. Soc. Indust. Appl. Math., v. 12, 1964, pp. 807-826. MR 33 \#5134.

4. D. J. Evans \& C. V. D. ForRINGTON, "An iterative process for optimizing symmetric overrelaxation," Comput. J., v. 6, 1964, pp. 271-273.

5. G. H. GolUB \& R. S. VARGA, "Chebychev semi-iterative methods, successive overrelaxation iterative methods, and second order Richardson iterative methods. I, II," Numer. Math., v. 3, 1961, pp. 147-168. MR 26 \#3207; 3208.

6. G. J. HABETLER \& E. L. WACHSPRESS, "Symmetric successive overrelaxation in solving diffusion difference equations," Math. Comp., v. 15, 1961, pp. 356-362. MR 23 \#B2176.

7. W. KAHAN, Gauss-Seidel Methods of Solving Large Systems of Linear Equations, Ph.D. Thesis, University of Toronto, 1958.

8. M. S. LYNN, "On the equivalence of SOR, SSOR, and USSOR as applied to $\sigma_{1}$-ordered systems of linear equations," Comput. J., v. 7, 1964, pp. 72-75. MR 31 \#4157.

9. Leland K. MCDowell, Variable Successive Overrelaxation, Report No. 244, Dept. of Computer Sciences, University of Illinois, September 18, 1967.

10. A. M. OsTROwSKI, "On the linear iteration procedures for symmetric matrices," Rend. Mat.e Appl., v. 14, 1954, pp. 140-163. MR 16, 1155.

11. J. W. SHELDON, "On the numerical solution of elliptic difference equations," Math. Tables Aids Comput., v. 9, 1955, pp. 101-112. MR 17, 668.

12. J. W. SHELDON, "On the spectral norms of several iterative processes," J. Assoc. Comput. Mach., v. 6, 1959, pp. 494-505. MR 23 \#B1647.

13. RicharD S. VARGA, "A comparison of the successive overrelaxation method and semiiterative methods using Chebychev polynomials," J. SIAM, v. 5, 1957, pp. 39-46. MR 19, 772.

14. E. L. WACHSPRESS, Iterative Solution of Elliptic Systems and Applications to the Neutron Diffusion Equations of Reactor Physics, Prentice-Hall, Englewood Cliffs, N. J., 1966.

15. David M. Young, "Iterative methods for solving partial difference equations of elliptic type," Trans. Amer. Math. Soc., v. 76, 1954, pp. 92-111. MR 15, 562.

16. David M. Young, MARY F. Wheeler \& JAMEs A. Downing, On the Use of the Modified Successive Overrelaxation Method with Several Relaxation Factors, Proc. IFIP 65, W. A. Kalenich (editor), Spartan Books, Washington, D. C., 1965.

17. David M. Young \& DAvid R. KINCAID, Norms of the Successive Overrelaxation Method and Related Methods, Computation Center Report TNN-94, University of Texas, Austin, September 1969.

18. David M. Young, Convergence Properties of the Symmetric and Unsymmetric Successive Overrelaxation Methods and Related Methods, Computation Center Report TNN-96, University of Texas, Austin, September 1969. 